\title{
THE PHASE DIAGRAM OF THE SECOND-TYPE ANTIFERROMAGNET
}

\author{
T. Balcerzak and A. Radomska \\ Department of Solid State Physics, University of Eódź \\ Pomorska 149/153, 90-236 Łódź, Poland
}

\begin{abstract}
We investigate theoretically the phase diagrams of the second-type antiferromagnet with the fcc structure. Besides the nearest-neighbour and next-nearest-neighbour exchange interactions, the biquadratic and long-range dipolar terms are taken into account. The results can be useful to discuss the phase diagram of europium telluride under external pressure.

PACS numbers: 75.10.-b, 75.50.Ee, 75.50.Pp
\end{abstract}

\section{Introduction}

Europium telluride, EuTe, is a well-known example of the fcc second-type antiferromagnet with $S=7 / 2[1,2]$. Its former theoretical description has been given within the Heisenberg model with nearest-neighbour (nn) and next-nearest-neighbour (nnn) exchange interactions taken into account. At normal pressure, the nn interaction, $J_{1}$, is known to be positive and about three times weaker than the nnn one, $J_{2}$, which is negative. The resulting spontaneous magnetisation shows ferromagnetic alignment of spins within (111) plane and the antiferromagnetic arrangement of adjacent planes.

Recently, for such a system, the role of biquadratic interactions has been pointed out $[3,4]$, as well as the role of long-range dipolar interactions [5]. The existence of such terms, which are usually not taken into account with the Heisenberg Hamiltonian, became a stimulus for the present studies. Namely, the aim of our work is to investigate the influence of biquadratic and dipolar interactions on the phase diagrams of the second-type antiferromagnet.

To explain the method, the outline of the theory will be given in the next section. The numerical results and discussion will be presented in the last section. 


\section{Theory}

The Hamiltonian of the system can be written in the form:

$$
\begin{aligned}
\mathcal{H}= & -J_{1} \sum_{n n} S_{i} S_{j}-J_{2} \sum_{n n n} S_{i} S_{j}-A \sum_{\substack{n n \\
n n n}}\left(S_{i} S_{j}\right)^{2}+g \mu_{\mathrm{B}} H^{z} \sum_{i} S_{i}^{z} \\
& +\frac{\left(g \mu_{\mathrm{B}}\right)^{2}}{2} \sum_{i, j} \frac{1}{r_{i, j}^{3}}\left[S_{i} S_{j}-3 \frac{\left(S_{i} r_{i, j}\right)\left(S_{j} r_{i, j}\right)}{r_{i, j}^{2}}\right],
\end{aligned}
$$

where $S=7 / 2, J_{1}$ and $J_{2}$ are the exchange integrals for nn and nnn interactions, respectively, whereas $A$ describes the strength of biquadratic interactions, both $n n$ and $n n n$. The fourth term in the Hamiltonian is the Zeeman term which includes the external field $H z$, while the last term describes the long-range dipolar interactions.

Taking into account the relative complexity of the system, for further studies we have adopted a simple theory, namely the molecular field approximation (MFA) with autocorrelations. Then, within the MFA method, the Gibbs energy for the Hamiltonian (1) can be calculated, and hence all thermodynamic properties of the system can be found. The long and tedious derivations, however, will not be presented in this contribution. In particular, we obtained the phase transition temperature, from the ordered to paramagnetic state, in the following form:

$$
k_{\mathrm{B}} T=2 q\left[6 J_{1}-\alpha \pm 6\left(J_{1}+J_{2}\right)\right],
$$

where "-" or "+" corresponds to the Néel or Curie temperature, respectively. Here, $\alpha$ is the parameter corresponding to dipolar interactions, namely

$$
\alpha=\frac{\left(g \mu_{\mathrm{B}}\right)^{2}}{2} \sum_{j} \frac{1}{r_{i, j}^{3}}\left[1-3\left(\frac{z_{i, j}}{r_{i, j}}\right)^{2}\right]
$$

and $q=\left\langle\left(S_{i}^{z}\right)^{2}\right\rangle$ is the quadrupolar moment. The explicit expression for $q$ is of the following form:

$$
q=\frac{1}{4}\left\{\sum_{n=0}^{3}(2 n+1)^{2} \exp \left[\frac{(2 n+1)^{2}}{4} x\right]\right\} /\left\{\sum_{n=0}^{3} \exp \left[\frac{(2 n+1)^{2}}{4} x\right]\right\}
$$

where

$$
x=18 A /\left[6 J_{1}-\alpha \pm 6\left(J_{1}+J_{2}\right)\right] .
$$

It is easy to check that if we neglect the dipolar interactions $(\alpha=0)$, as well as quadrupolar interactions $(A=0)$, then our general formula (2) reduces to that for the Néel and Curie temperatures, known from the previous works $[1,2]$.

\section{The numerical results and discussion}

Under normal pressure, EuTe is the second-type antiferromagnet. The experimental values of the Néel temperature $\left(T_{N}\right)$ and asymptotic Curie temperature $\left(T_{\mathrm{C}}\right)$ are known merely approximately: $T_{\mathrm{N}} \approx 9.6 \mathrm{~K}$ and $T_{\mathrm{C}} \approx-4 \mathrm{~K}$ [2]. Assuming these values, and putting in the first approximation $\alpha=0$ and $A=0$, we get from 
Eq. (2) the values $J_{1} / k_{\mathrm{B}}=0.044 \mathrm{~K}$ and $J_{2} / k_{\mathrm{B}}=-0.152 \mathrm{~K}$. When the biquadratic interaction (with the value $A / J_{2}=1.5 \times 10^{3}[4]$ ) and the dipolar interactions (with the value $\alpha / k_{\mathrm{B}}=-0.068 \mathrm{~K}[5]$ ) were taken into account, we obtained the corrected values for the exchange integrals, namely $J_{1} / k_{\mathrm{B}}=0.036 \mathrm{~K}$ and $J_{2} / k_{\mathrm{B}}=-0.144 \mathrm{~K}$. All these values (within accuracy of the experimental errors) approximately agree with those given in the literature $[2,5]$; one has to note, however, that for such estimations the exact knowledge of $T_{\mathrm{N}}$ and $T_{\mathrm{C}}$ is crucial.

When calculating the phase diagram we are aware of the fact that for EuX compounds (X $=\mathrm{O}, \mathrm{S}, \mathrm{Se}, \mathrm{Te})$ both $J_{1}$ and $J_{2}$ depend on the lattice constant [1]. On the other hand, for a given compound, the lattice constant can be changed using the high external pressure. For instance, the recent experiment performed for EuTe showed that at the external pressure above $9 \mathrm{GPa}$ the ferromagnetic phase is established [6].

The obtained phase diagrams (Figs. 1 and 2) are the Néel and Curie temperatures vs. $J_{1}$ and $J_{2}$. The stability of all possible phases, i.e., antiferromagnetic (AF), ferromagnetic $(F)$, and paramagnetic $(P)$, has been checked by calculations of the Gibbs energy. The transition between $\mathrm{AF}$ and $\mathrm{F}$ phases (the dashed line drawn in the figures at $\left.J_{1}=\left|J_{2}\right|\right)$ is of the first order. It is worth noticing that $T_{\mathrm{N}}$ does not depend on $J_{1}$ (Fig. $1 \mathrm{a}$ and $\mathrm{b}$ ) but it depends on $J_{2}$ (Fig. $2 \mathrm{a}$ and b), whereas $T_{\mathrm{C}}$ depends linearly on both $J_{1}$ and $J_{2}$. In Figs. 1a and 2 a the influence of dipolar interactions on phase transition temperature is shown. As a result, an increase in both $T_{\mathrm{N}}$ and $T_{\mathrm{C}}$ is observed. On the other hand, in Figs. $1 \mathrm{~b}$ and $2 \mathrm{~b}$ we plotted the transition temperature for different values of the biquadratic interaction parameters (negative or positive). As we can see, the change of the sign from negative to positive $A$ results in the increase in phase transition temperature. Other

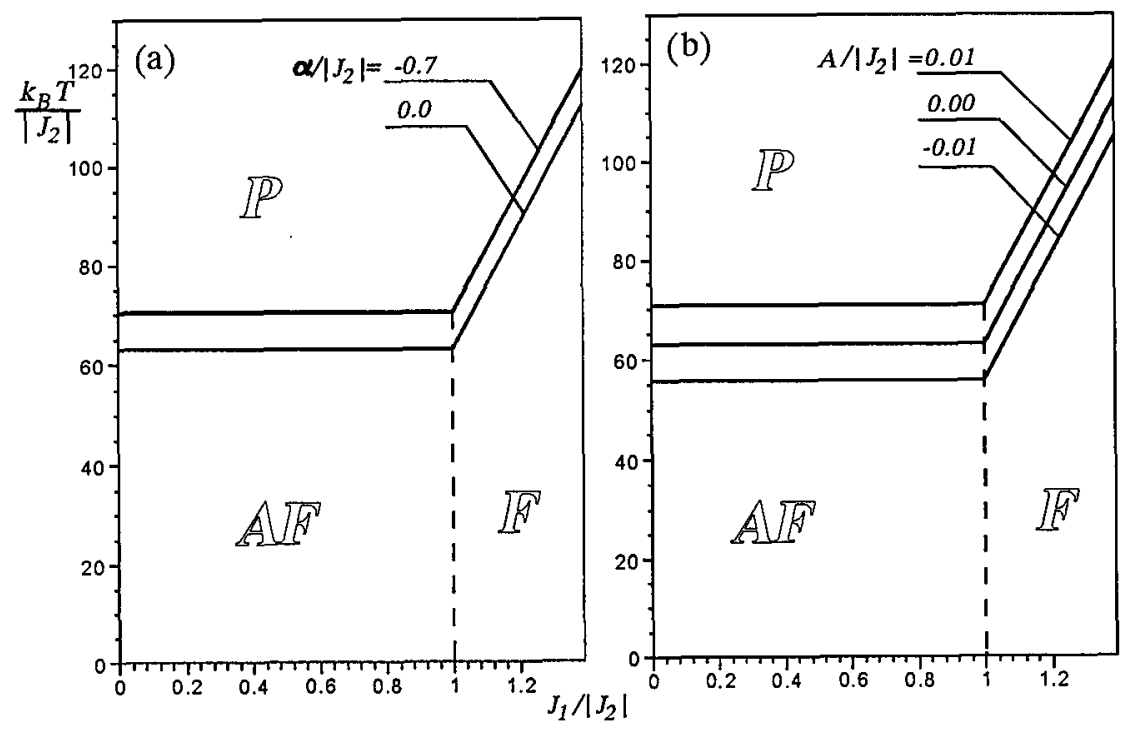

Fig. 1. The phase diagram, $k_{\mathrm{B}} \mathrm{T} /\left|\mathrm{J}_{2}\right|$ vs. $J_{1} /\left|J_{2}\right|$, (a) for $A=0$, (b) for $\alpha=0$. 

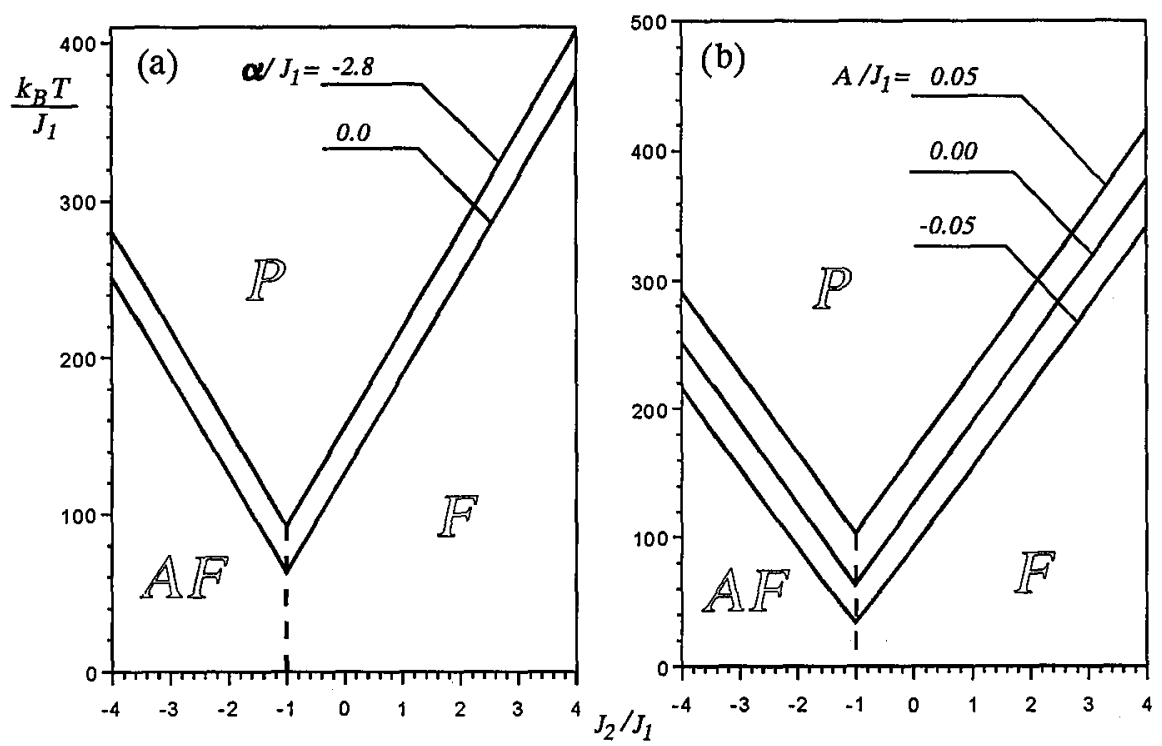

Fig. 2. The phase diagram, $k_{\mathrm{B}} T / J_{1}$ vs. $J_{2} / J_{1}$, (a) for $A=0$, (b) for $\alpha=0$.

phase diagrams, for instance the Néel (Curie) temperatures vs. $A$, have also been calculated, but they are not presented in this paper.

In conclusion, the results of the present work demonstrate the influence of biquadratic and dipolar interactions on the phase diagram of the second-type antiferromagnet. The structure of our diagrams (especially Fig. 1a and b) confirms qualitatively the phase diagram obtained experimentally for EuTe [6]. However, in order to describe the phase transition temperature vs. lattice constant $a$ (as it was experimentally found in Ref. [6]), the detailed dependence of $J_{1}(a), J_{2}(a)$, and $A(a)$ should be known for a given compound. A continuation of such studies is planned as our future work.

\section{References}

[1] W.P. Wolf, T.R. McGuire, M.W. Shafer, J. Appl. Phys. 35, 984 (1964).

[2] W. Zinn, J. Magn. Magn. Mater. 3, 23 (1976).

[3] U. Köbler, R. Mueller, L. Smardz, D. Maier, K. Fisher, B. Olefs, W. Zinn, Z. Phys. B 100, 497 (1996).

[4] J.J. Chen, G. Dresselhaus, M.S. Dresselhaus, G. Springholz, C. Pichler, G. Bauer, Phys. Rev. B 54, 402 (1996).

[5] L. Bergomi, J.J. Chen, Phys. Rev. B 56, 3281 (1997).

[6] M. Ishizuka, Y. Kai, R. Akimoto, M. Kobayashi, K. Amaya, S. Endo, J. Magn. Magn. Mater. 166, 211 (1997). 